\title{
IN-SITU INVESTIGATION of PLASTICITY at NANO-SCALE
}

\author{
Julia R. Greer", Ju-Young Kim \& Steffen Brinckmann \\ "Department of Materials Science, California Institute of Technology, Pasadena, CA, 91125, USA
}

\begin{abstract}
$\underline{\text { Summarv }}$
Mechanical behavior of crystals is dictated by dislocation motion in response to applied force. While it is extremely difficult to directly observe the motion of individual dislocations, several correlations can be made between the microscopic stress-strain behavior and dislocation activity. Here, we present for the first time the differences observed between mechanical behavior in two fundamental types of crystals: face-centered cubic, fcc ( $\mathrm{Au}, \mathrm{Cu}, \mathrm{Al}, \mathrm{Ni}$, etc.) and body-centered cubic, bcc (W, $\mathrm{Cr}, \mathrm{Mo}, \mathrm{Nb}$, etc.) with sub-micron dimensions subjected to in-situ micro-compression in SEM chamber. In a striking deviation from classical mechanics, there is a significant increase in strength as crystal size is reduced to $100 \mathrm{~nm}$; however in gold crystals (fcc) the highest strength achieved represents $44 \%$ of its theoretical strength while in molybdenum crystals (bcc) it is only $7 \%$. Moreover, unlike in bulk where plasticity commences in a smooth fashion, both nano-crystals exhibit numerous discrete strain bursts during plastic deformation. These remarkable differences in mechanical response of fce and bcc crystals to uniaxial micro-compression challenge the applicability of conventional strain-hardening to nano-scale crystals. We postulate that they arise from significant differences in dislocation behavior between fcc and bcc crystals at nanoscale and serve as the fundamental reason for the observed differences in their plastic deformation. Namely, dislocation starvation is the predominant mechanism of plasticity in nano-scale fcc crystals while junction formation and subsequent hardening characterize bcc plasticity, as confirmed by the microstructural electron microscopy. Experimentally obtained stress-strain curves together with video frames during deformation and cross-sectional TEM analysis are presented, and a statistical analysis of avalanche-like strain bursts is performed for both crystals and compared with stochastic models.
\end{abstract}

In this work we investigate flow stress as a function of diameter in several fec metals (gold, aluminum, and copper) and bec metals (molybdenum, niobium, and tungsten) single crystal nano-pillars subjected to uniaxial micro-compression. The results that follow suggest that fcc and bec crystals have fundamentally different plasticity mechanisms when reduced to nano-scale with significant strain-hardening present in the latter and virtually none in the former. The attainment of nearly $50 \%$ of the theoretical strength in Au suggests that plasticity is likely controlled by nucleation of new dislocations rather than by interactions of the pre-existing ones. On the contrary, the smallest Mo nano-pillar achieves only $\sim 7 \%$ of its theoretical strength, implying that plasticity is likely driven by the intricate motion and interactions of dislocations inside the pillar rather than by nucleation events.

Single crystal nano-pillars described in this work were fabricated via Focused Ion Beam (FIB) and subsequently uniaxially compressed along $<001>$ direction with a flat punch tip of $30 \mu \mathrm{m}$ diameter. The specifics of nano-pillar fabrication are based on the authors' previous work [1], however, unlike previously reported, the testing was performed in-situ in "SEMentor," a one-of-a-kind instrument, which combines the strengths of SEM (for visualization) and Nanoindenter (precise loading control). SEM images of gold (initial diameter $=185 \mathrm{~nm}$ ) nano-pillar before and after compression showing homogeneous deformation are shown in Fig. 1(a) and (b), and a compressed Mo pillar with clear multiple crystallographic slip lines is shown in Fig. 1(c).
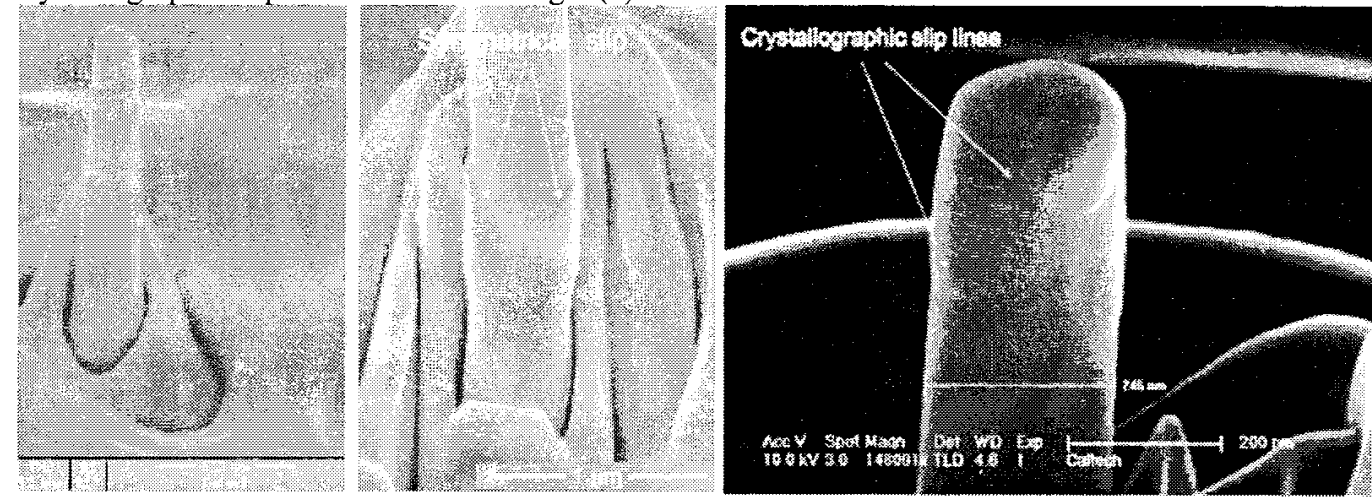

Figure 1. (a) Au $<001>$-oriented pillat before compression with initial diameter of $185 \mathrm{~nm}$

(b) Mid-section of a representative gold pullar after compression showing symmetric donble-slip.

(c) Mo <001>-oriented pilfar with intial diameter of $205 \mathrm{~nm}$ after compression ( $\$ 2^{\circ}$ tilt angle for all).

Load-displacement data collected during the compressions were subsequently converted into uniaxial stress-plastic strain relationship, and some of the representative curves for both crystals are shown in Fig. 2. 

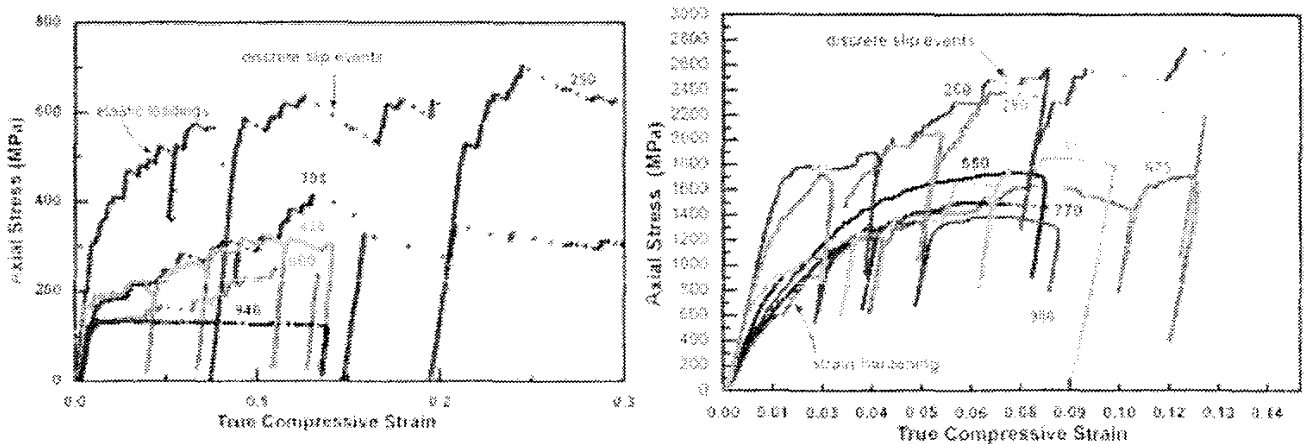

Figure 2. Some representative stress vs. strain curves for (a) fcc Au and (b) bce Mo nano-pillars. Some plllars were intentionally unloaded and re-loaded several times.

An interesting feature of these curves is that unlike in a typical smooth stress-strain plot of bulk single crystals, the stress-strain relationship for smaller pillars does not undergo Stage II hardening, but instcad is composed exclusively of elastic loading segments followed by discrete displacement bursts. These bursts are likely correlated with the initiation of dislocation avalanches, activated in response to the shear stress in the glide plane. Contrary to the very discontinuous stress-strain behavior in gold, the results for molybdenum nanopillars subjected to the same uniaxial deformation are drastically different, as shown in Fig. 2(b). In the early stages of post-yield deformation clear strain-hardening observed for most curves, which is manifested through the non-linearity of the stress vs. strain relationship. Interestingly, larger pillars exhibit a smoother and much more continuous deformation compared with smaller pillars, which show several discrete bursts throughout the process. The scaling relationship

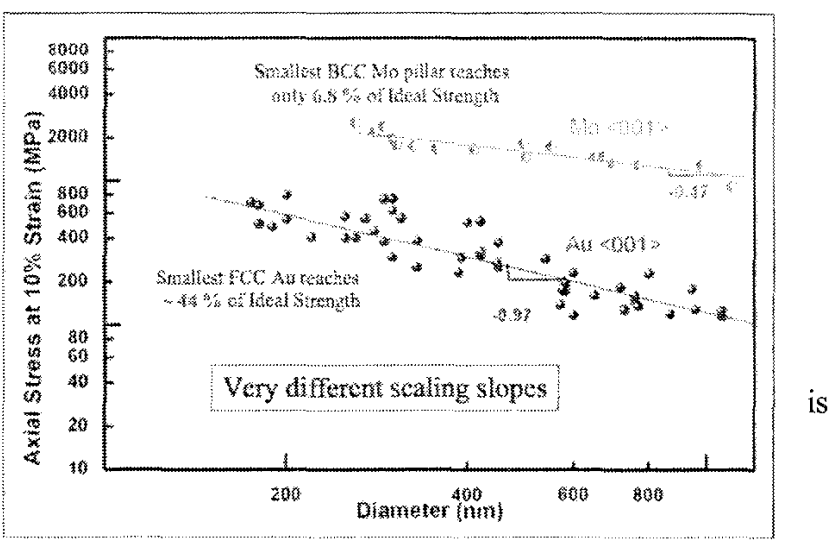

Figure 3. Log $10 g$ plot of llow stress as a function of initial diameter representing scaling laws for Mo and $\mathrm{Au}$. The strengthening siope for gold (foc) is $-2 x$ higher than that for molybdenum (boc). between attained stresses at a particular strain and diameter for both types of crystals clearly shows a power-law type dependence, as can be seen in the log-log plot in Fig. 3. While both crystals exhibit a power-law trend, the scaling slopes are vastly different: -0.97 for gold and -0.47 for molybdenum, indicating that the size effect is much more pronounced in fcc crystals compared with bcc. In addition, the smallest (205nm) Mo nano-pillar reaches the stress of $2.85 \mathrm{GPa}$ ( $7 \%$ of theoretical strength) compared with $\sim 800 \mathrm{MPa}$ ( $44 \%$ of ideal strength) for a $200 \mathrm{~nm}$ Au at equivalent strain. These nano-pillars can sustain strengths much higher than their bulk equivalents, of $\sim 450 \mathrm{MPa}$ for $\mathrm{Mo}[2]$ and $25 \mathrm{MPa}$ for $\mathrm{Au}[3]$.

These findings suggest that fundamentally different dislocation motion mechanisms might be operating in fcc and bcc crystals at nano-scale. The experimental results reported here are consistent with recent computational findings by molecular dynamics (MD) and 2-D and 3-D dislocation dynamics (DD) simulations [4-6]. Moreover, post-mortem cross-sectional TEM analysis of the deformed pillars reveals that Au crystals are nearly free of dislocations while Mo has ubiquitous dislocation loops concentrated primarily near the surface. Avalanche-like displacement burst behavior prevalent in all deformation curves is found to be independent of crystal structure and of specimen size, however the probability of slip events smaller than $\sim 10$ Burgers vectors is found to be lower than previously predicted.

\section{References}

[1] Greer JR, Oliver, W.C., Nix, W.D. Size Dependence of Mechanical Properties of Gold at the Micron Scale in the Absence of Strain Gradients. Acta Materialia 2005;53:1821.

[2] F. Guiu PLP. The effect of orientation on the yielding and flow of molybdenum single crystals. Physica Status Solidi 1966;15:539.

[3] Savitskii EM. Handbook of Precious Metals: Hemisphere Publishing Company, 1969.

[4] Balint DS, Deshpande, V.S., Needleman, A., Van der Giessen, E. Size effects in uniaxial deformation of single and polycrystals: a discrete dislocation plasticity analysis. Modeling and Simulation in materials Science and Engineering 2006;14:409.

[5] Chang J, Cai, W., Bulatov, V.V., Yip, S. Dislocation motion in BCC metals by molecular dynamics Materials Science and Engineering A 2001;309-310:160.

[6] Espinosa HD, Panico, M., Berbenni, S., Schwartz, K.W. Discrete Dislocation Dynamics simulations to interpret plasticity size and surface effects in freestanding fcc thin films. International Journal of Plasticity 2006;22:2091. 